\title{
COMUNICACIÓN
}

\section{ECTOPARÁSITOS EN CANINOS DE LOS DISTRITOS DE LA ZONA CLIMÁTICA NORTE DE LIMA METROPOLITANA}

\author{
Luis Estares P. ${ }^{1}$, Amanda Chávez V. ${ }^{2}$ y Eva Casas A. ${ }^{2}$
}

Resumen

\begin{abstract}
A total of 400 randomly selected male and female dogs from 1 to 16 months of age were studied to determine ectoparasite prevalence in the San Juan de Lurigancho, San Martín de Porres, Comas and Independencia districts of northern Lima. Sampling took place during the austral summer, December 1998 to February 1999, when ambient temperature and relative humidity varied between $18-26.7^{\circ} \mathrm{C}$ and $79-99 \%$. Ectoparasite prevalence was extremely elevated, reaching $100 \%$ in Independencia, $99.0 \%$ in Comas, 98.2\% in San Martín de Porras and 98.7\% in San Juan de Lurigancho. The most common ectoparasites were fleas (Ctenocephalides felis felis $89.0 \% \pm 3.1$ and Pulex irritans $37.8 \% \pm 4.8$ ), followed by ticks (Rhipicephalus sanguineus $30.0 \% \pm 4.5$ ), lice (Heterodoxus spiniger $9.3 \% \pm 2.8$ ) and mites (Demodex canis 3.8\% \pm 1.9 ). The extensive presence of fleas, especially $C$. felis felis, likely indicates that the elevated incidence of dermatological problems observed in the majority of the dogs studied is an allergic dermatitis produced by flea bites.
\end{abstract}

Key words: Dog ectoparasites, fleas, ticks, lice, mites.

Palabras clave: Ectoparásitos, perros, pulgas, garrapatas, ácaros.

Los agentes etiológicos del parasitismo externo canino son causantes de múltiples problemas, tanto para el animal como para los dueños y personas cercanas a ellos, siendo uno de los problemas asociados con la tenencia de animales, la dermatitis alérgica, presentándose cuadros dramáticos en personas hipersensibles. Por otro lado, las picaduras de las pulgas (Ctenocephalides felis felis) pueden ocasionar dermatitis alérgica, pudiéndose también transmitir a tra-

\section{Práctica privada}

2Laboratorio de Parasitología IVITA - FMV UNMSM.E.mail:d1700010@unmsm.edu.pe. vés de las pulgas ( $C$. felis felis y Pulex irritans), otros agentes, como la Yersinia pestis; además, los piojos y pulgas son los hospederos intermediarios del Diphylidium caninum.

La infestación en caninos, depende de varios factores entre ellos el estado de alimentación, estado inmunológico, carga parasitaria, así como la temperatura y humedad del medio ambiente. La temperatura es el factor intrínseco más importante que influye sobre la existencia de los parásitos y el desarrollo de sus procesos vitales, los cuales pueden verse incrementados en relación directa a la temperatura y humedad (Brack, 1976). Bajo este punto de vista, 
Quezada (1987) realizó un estudio sobre el confort climático en Lima Metropolitana y Callao, dividiendo el área en cuatro zonas climáticas; litoral, caliente, norte y sur, es así que bajo este contexto surgió la necesidad de realizar estudios de ectoparásitos en las diferentes zonas climáticas.

El presente trabajo tuvo como objetivo determinar las prevalencias de los principales ectoparásitos en caninos de la zona climática norte de Lima Metropolitana durante la estación de verano.

Se muestrearon al azar 400 perros de ambos sexos, cuyas edades oscilaban entre 1 mes y 16 años con mediana de 1 año. Las muestras provinieron de los distritos de San Juan de Lurigancho, San Martín de Porres, Comas e Independencia durante los meses de diciembre de 1998 a febrero de 1999. La temperatura ambiental en dicho periodo estuvo entre $18^{\circ} \mathrm{C}$ y $26.7^{\circ} \mathrm{C}$ con una humedad relativa entre $70 \%$ y $99 \%$.

Los parásitos externos fueron extraídos de todo el cuerpo del animal según las técnicas de Melhorn y Duwel (1993) y Soulsby (1988). La identificación se realizó en el Laboratorio de Parasitología de la Facultad de Medicina Veterinaria, UNMSM.

La prevalencia de parasitismo externo en perros de la zona climática norte de Lima fue de $98.8 \% \pm 1.1$, siendo el valor muy similar para los cinco distritos de la zona como se muestra en el Cuadro 1. Estudios anteriores realizados en 100 perros reportaron un porcentaje total de $91.0 \%$ (Acha, 1952), posteriormente Liberato (1998) examinando 400 perros de la zona climática sur en la estación de verano, encontró una prevalencia promedio de $85.5 \% \pm 3.45$ y Bustamante (1998) en 390 perros de la zona climática Litoral en invierno halló una prevalencia de $94.4 \% \pm 2.3$.

Entre pulgas, garrapatas y ácaros se pudo identificar nueve especies de ectoparásitos en este estudio (Cuadro 2), a diferencia de Acha (1952) que sólo identificó 5 especies, mientras que Liberato (1998) y Bustamante (1998) identificaron 8 y 9 especies respectivamente.

El aumento de especies reportadas recientemente, podría obedecer a la migración hacia la capital en las últimas décadas, incorporando otras formas de vida y costumbres. Así, se tiene la presencia de la pulga de la gallina Echidnophaga gallinacea en el perro. Además, no se puede descartar la importación de nuevos parásitos, como el caso de la garrapata parda del perro, el Rhipicephalus sanguineus, originaria del África y presente en nuestros caninos; así mismo tenemos el caso del piojo Heterodoxus spiniger originario de Australia (Georgi y Georgi, 1996).

Las especies de ectoparásitos de mayor prevalencia en perros lo constituyen las pulgas, principalmente el Ctenocephalides felis felis $(89.0 \% \pm 3.1)$, seguida por el Pulex irritans $(37.8 \% \pm$ 4.8 ), concordando lo hallado en USA por Dryden y Rust (1994). Por otro lado, Chukwu, 1985 reportó en el Estado de Arambra (Nigeria) C. canis en un $26.3 \%$ y E. gallinacea en un $2.1 \%$. Koutinas $e t$ al. (1995), en Grecia, señaló que el $C$. canis es la especie más común en perros (71\%), seguida del C.felis felis $(40.3 \%)$.

En general, todas las especies de pulgas identificadas en el presente estudio son cosmopolitas. El C. felis felis es la pulga más frecuente en muchas zonas del planeta, pero se ve desplazada esporádicamente por otras sub especies 
Cuadro 1. Prevalencia de parasitismo externo en perros por distritos de la zona climática norte de Lima Metropolitana en la estación de verano.

\begin{tabular}{lccc}
\hline Distritos & $\begin{array}{c}\text { Perros } \\
\text { examinados, } \\
n\end{array}$ & $\begin{array}{c}\text { Perros } \\
\text { parasitados, } \\
\text { ndependencia }\end{array}$ & $\begin{array}{c}\text { Prevalencia de } \\
\text { parásitos } \\
(\%)+\mathrm{IC}\end{array}$ \\
\hline Comas & 48 & 48 & 100.0 \\
San Martín de Porres & 104 & 103 & $99.0 \pm 1.9$ \\
San Juan de Lurigancho & 98 & 96 & $98.2 \pm 2.0$ \\
\hline TOTAL & 150 & 148 & $98.7 \pm 1.8$ \\
\hline
\end{tabular}

Cuadro 2. Tipo y prevalencia de parásitos externos en perros de la zona climática norte de Lima Metropolitana en la estación de verano.

\begin{tabular}{lcc}
\hline Parásito & $\begin{array}{c}\text { Perros parasitados, } \\
\mathrm{n}\end{array}$ & $\begin{array}{c}\text { Prevalencia de } \\
\text { parasitismo } \\
\text { externo, \% } \pm \text { IC }\end{array}$ \\
\hline Pulgas & & \\
Ctenocephalides felis felis & 356 & $89.0 \pm 3.1$ \\
Ctenocephalides canis & 7 & $1.8 \pm 1.3$ \\
Pulex irritans & 151 & $37.8 \pm 4.8$ \\
Echidnophaga gallinacea & 10 & $2.5 \pm 1.5$ \\
Piojos & 1 & $0.3 \pm 0.5$ \\
Linognathus setosus & 37 & $9.3 \pm 2.8$ \\
Heterodoxus spiniger & 120 & $30.0 \pm 4.5$ \\
Garrapata & & $0.5 \pm 0.7$ \\
Rhipicephalus sanguineus & 2 & $3.8 \pm 1.9$ \\
Ácaros & 15 & \\
Sarcoptes scabiei & & \\
Demodex canis & & \\
\hline
\end{tabular}

en determinadas regiones. Georgi y Georgi (1996) señala que el C. canis es menos común que el $C$. felis felis debido a que la primera se restringe unicamente a cánidos, mientras que la segunda, debido a su poca afinidad por los hospederos, puede infestar a un gran rango de especies domésticas y silvestres, incluyendo las ratas, lo que favorece su difusión.
En relación a salud pública, las personas hipersensibles sufren los estragos por las picaduras de las pulgas, así también, éstas pueden transmitir la peste (Yersenia pestis), posiblemente a través de infestaciones por Pulex spp; además pueden presentar cuadros de gastroenteritis, causados por D. caninum adquirido por el hábito de espulgar a sus perros (Leguía, 1996). 
Al igual que el hombre, los perros también presentan cuadros de dermatitis alérgica por picadura de pulgas (DAPP). Mercado (1993), encontró que aproximadamente la quinta parte de los animales que llegan a consulta presentan problemas de piel, y de ellos la mayor frecuencia la constituye la DAPP, siendo la pulga de mayor prevalencia el $C$. felis felis y probablemente la principal agente causante de DAPP en Lima.

La prevalencia del $R$. sanguineus fue de $30 \% \pm 4.5$; similar al obtenido por Liberato (1998) en la zona climática sur en verano $(11.75 \% \pm 9.2)$. Al respecto, Horak (1982) determinó la prevalencia estacional del $R$. sanguineus en un criadero de perros, encontrando la mayor carga parasitaria durante el inicio y mediados de la estación de verano. Sin embargo, Bustamante (1998) realizó un estudioen la zona climática Litoral en invierno, encontrando una prevalencia de $2.8 \% \pm 1.6$. Se considera que la temperatura y humedad adecuada favorecen el ciclo biológico de parásito haciéndolo más corto. Por otro lado, Inokuma et al. (1996) encontraron que en un criadero de perros en Japón, las garrapatas desaparecieron del cuerpo de los animales cuando la temperatura fue menor a $15^{\circ} \mathrm{C}$.

El piojo más común fue el $H$. spiniger $(9.3 \% \pm 2.8)$, similar a lo hallado en la zona climática litoral y sur. Este parásito ataca principalmente a animales jóvenes y débiles causando irritaciones dérmicas, intranquilidad y deterioro de pieles (Bustamante, 1998; Liberato, 1998).

\section{Literatura Citha}

1. Acha, P. 1952. Porcentaje de parásitos del Canis familiaris en la ciudad de Lima. Tesis Bach. Fac. Med. Vet.
UNMSM. Lima. 35p.

2. Brack, E. 1976. Ecología Animal. Universidad Nacional Agraria. Dpto..Biología: 113.

3. Bustamante, A. 1998. Prevalencia de ectoparásitos en Canis familiaris en la zona climática Litoral de Lima metropolitana en la estación de invierno. Tesis Med. Veterinario. Fac. Med. Vet. UNMSM. Lima. 43p.

4. Chukwu, C. 1985. Prevalence of fleas on dogs in Anambra State of Nigeria. Journal Zoonoses 12(3): 192-195.

5. Dryden, M.W. y M. Rust. 1994. Veterinary Parasitology. Ed. Continental. México. 52(94): 1- 19.

6. Georgi, J. y M. Georgi. 1996. Parasitología en clínica canina. Ed. Interamericana. México. $231 \mathrm{p}$.

7. Horak, I. 1982. Parasites of domestic and wild animals in South Africa. The seasonal prevalence of $R$. sanguineus and Ctenocephalides spp. on kenelled dogs in Pretoria North. Onderspoort J. Vet. 49(1): 63-68.

8. Inokuma, H.; K. Tamura y T. Onishi. 1996. Seasonal ocurrence of $R$. sanguineus in Okayama prefecture, Japan and effect of temperature of development of the tick. Journal Vet Med Sci. 3(58): 225-228.

9. Koutinas, A.; M. Parazahariadou y T. Rallis. 1995. Flea species from dogs and cats in Northem Greece, enviromental and clinical implications. Vet. Parasitology 1-2 (58).

10. Leguía, P. 1996. Enfermedades parasitarias de perros y gatos. Edit. de Mar. Lima, Perú. $127 \mathrm{p}$.

11. Liberato, W. 1998. Prevalencia de ectoparásitos en Canis familiaris en los distritos de San Juan de Miraflores, Villa María del Triunfo y Villa el Salvador. Tesis Med. Veterinario. Fac. Med. Vet. UNMSM. Lima. 21p. 
12. Melhorn, M. y D. Duwel. 1993. Manual de Parasitología Veterinaria. Ed. Presencia Ltda. Colombia. 436 p.

13. Mercado, S. 1993. Incidencia de las dermatopatías en caninos durante el período de 1981-1990 de los estratos socioeconómicos medio y alto del área de Lima Metropolitana. Tesis de Med. Vete- rinario. Fac. Med. Vet. UNMSM. 48 p.

14. Quezada, P. 1987. Estudio del confort ciimático en Lima Metropolitana y Callao. Fac. Ciencias. UNAM. 130 p.

15. Soulsby, E. 1988. Parasitología y enfermedades parasitarias en los animales domésticos. $7 \mathrm{ma}$ ed. Editorial Interamericana. México. $823 \mathrm{p}$. 